\title{
What Physical World Do We Live in?
}

\author{
Alexander Alexandrovich Antonov \\ Research Center of Information Technologies “TELAN Electronics”, Kiev, Ukraine \\ Email: telan@bk.ru
}

How to cite this paper: Antonov, A.A. (2016) What Physical World Do We Live in? Journal of Modern Physics, 7, 1933-1943. http://dx.doi.org/10.4236/jmp.2016.714170

Received: July 3, 2016

Accepted: October 22, 2016

Published: October 25, 2016

Copyright $\odot 2016$ by author and Scientific Research Publishing Inc. This work is licensed under the Creative Commons Attribution International License (CC BY 4.0).

http://creativecommons.org/licenses/by/4.0/ (c) (i) Open Access

\begin{abstract}
It is shown that the hypothesis of Monoverse relevant to the existing version of the special relativity theory is incorrect. Incorrect is also the existing version of the special relativity theory itself. Its revised version, the relativistic formula of which allows to propose the hypothesis of hidden Multiverse is presented. The hypothesis of hidden Multiverse is based on a proof by the author general scientific principle of physical reality of imaginary numbers. It is shown that this hypothesis, unlike many other hypotheses of Multiverse and the hypothesis of Monoverse, is already verifiable. And the experimental proof of the reality of the hidden Multiverse existence is a phenomenon of dark matter and dark energy. Other possible experimental proof of that is proposed as well. As a result, the data processing is obtained by WMAP and Planck spacecrafts, and the structure of hidden Multiverse is determined, which has proved to be a quaternion, and its parameters are defined. According to these calculations, the hidden Multiverse contains from twenty to twenty-two parallel universes, five-six neighboring universes of which constitute dark matter, and the rest parallel universes constitute dark energy. Moreover, it is shown that our hidden Multiverse neighbors with other hidden Multiverses, inaccessible to our observations not only by electromagnetic but also by gravitational manifestations, which together form a Supermultiverse.
\end{abstract}

\section{Keywords}

Multiverse, Imaginary Numbers, Dark Matter, Dark Energy, Special Theory of Relativity

\section{Introduction}

Let us recall the opinion on this issue prevailing in modern science. It is as follows. Among two currently recognized basic physical theories, such as the theory of relativity and the quantum physics, only special theory of relativity (STR) is relevant to this issue [1] [2]. It claims that, in accordance with the principle of light speed non-exceedance, 
we live in a monoverse.

However, there is a different opinion: we live in a multiverse, as the principle of light speed non-exceedance has been refuted by the principle of physical reality of imaginary numbers [3]-[6].

Let us see who is right.

STR has always been criticized throughout its century-old history. Arguments of its opponents, especially in the first half of the twentieth century, were often politically oriented. This led to development in STR supporters of certain immunity to criticism, including to the correct scientific criticism. Therefore, it has led to the belief that, as STR is immaculately perfect, criticism thereof is unnecessary.

In fact, over time some controversial or even wrong provisions, which have become the subject of such criticism, have been revealed in STR, as well as in any other scientific theory. They should be discussed, since, according to Karl Raimund Popper [7], the author of the "open society" concept, the conflict of opinions in scientific theories is inevitable and is a prerequisite for science development.

Therefore, STR, as well as other theories, needs friendly criticism, and appropriate adjustment, which is discussed further. However, not everyone agrees with this statement for various reasons.

And that is not big surprise. It is known from practical psychology [8] that people are always much more inclined to protest than agree. Therefore, it is a common situation in the history of science.

Nobel Prize winner Max Planck wrote: " $A$ new scientific truth does not triumph by convincing its opponents and making them see the light, but rather because its opponents eventually die, and a new generation grows up that is familiar with it".

\section{Why Special Theory of Relativity Is Considered Incomprehensible?}

There is also a common opinion that the STR has been subject to criticism due to the fact that it is very complex and therefore criticized by those who have not taken the trouble to comprehend it. There are at least two simple explanations of such an opinion.

- The first explanation corresponds to the aphorism by Sir Winston Churchill "History is written by the victors". They do not need criticism.

- The second explanation corresponds to the natural reluctance of STR developers to recognize that they did not understand too well in their theory. However, they had to explain it. And it has actually been more difficult for readers to understand corresponding fragments of the STR.

In particular, the authors of the STR did not know how to explain the physical sense of imaginary numbers discovered over five hundred years ago. Four hundred years before, famous mathematicians and physicists could not explain their physical sense and had to admit this. Whereas, the authors of the STR could not afford to agree to the fact, as all relativistic formulas at superluminal speeds in their theory led to results measured 
by imaginary numbers. Therefore, this result had to be urgently explained one way or another. Otherwise, the STR would not get recognition.

The latest statement is to be explained more exactly. For this we use, for example, the Lorentz-Einstein formula

$$
m=\frac{m_{0}}{\sqrt{1-(v / c)^{2}}}
$$

where $m_{0}$ is the rest mass of a moving entity;

$m$ is the relativistic mass of a moving entity;

$v$ is the velocity of a physical entity;

$c$ is the speed of light.

As can be seen from the formula and its diagram depicted in Figure 1(a), mass $m$ of entity moving at sub-light speeds $v<c$ is measured by real numbers, whereas at superluminal speeds $v>c$ it is measured by imaginary numbers. However, no one could ever explain what imaginary mass (for example, $5 i$ kilograms) is.

It is therefore in our opinion that the developers of the STR should leave this situation unexplained until better times, as their predecessors did in the previous four hundred years. It is clear that no theory can explain everything.

Nevertheless, the authors of the STR did otherwise, having actually postulated the principle of light speed non-exceedance. There certainly was some logic in this choice, as it allowed overcoming the non-recognition barrier. It is so convenient: if the situation $v>c$ is impossible in physics, there is nothing to explain. However, unfortunately, justification of this postulate has been unconvincing. Therefore, the principle of light speed non-exceedance has subsequently appeared to be refuted [9].

In order to better understand why things turned out this way, let's recall how the principle of light speed non-exceedance was substantiated in the early twentieth century. At that time (as well as currently) it was argued that, since relativistic mass, as follows from Formula (1), becomes indefinitely great, i.e. $\lim _{v \rightarrow c} m(v)=\infty$ when its speed approaches to the speed of light, breaking of light speed barrier needs infinitely great energy. However, this is impossible.

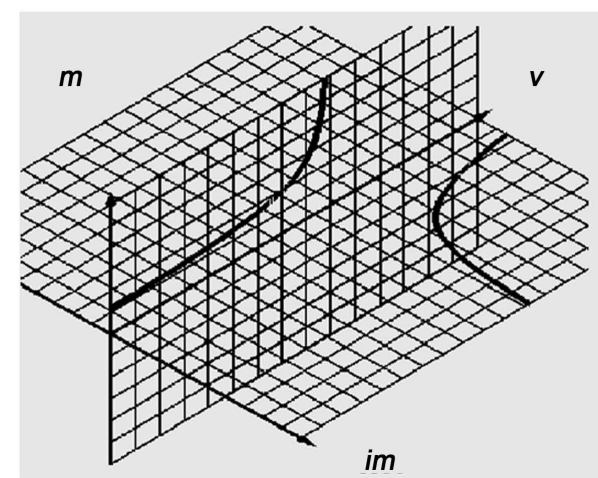

(a)

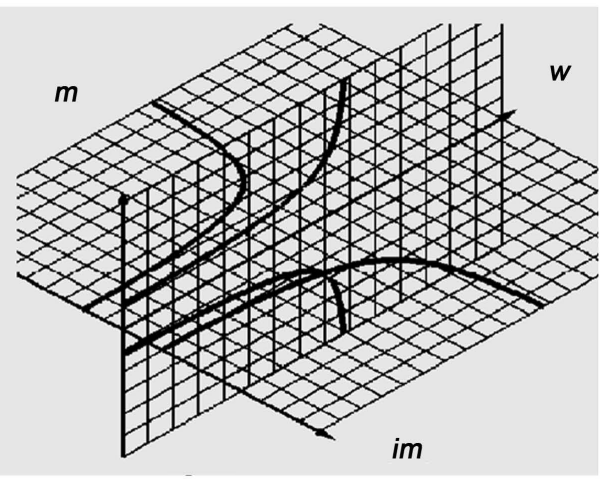

(b)

Figure 1. Graphs of functions (1) and (2). 
At first sight, everything seems to be right. But it is wrong impression. In fact, this argument is refuted even at the mundane level. Indeed, if you cannot, for example, get into the adjacent room through the wall of your house, it does not mean that you cannot get into this room otherwise (through the door) and that the room does not exist. This aspect was not taken into account in the STR, as there should necessarily be the ability to approve that no other physical world existed at $v>c$ (for not to explain it). In other words, one error (the principle of light speed non-exceedance) entailed another one (assertion of monoverse existence). Therefore, the STR still argues that we live in a monoverse.

Nevertheless, these arguments of the STR turned out to be its fundamental mistake, since the principle of light speed non-exceedance, as shown below, was refuted, after all. It took place due to the fact that physical reality of imaginary numbers was proved [3]-[6] in most indisputable way.

\section{How Has Physical Reality of Imaginary Numbers Been Proved?}

It was not even proved intentionally. There were just such circumstances. The fact was that in the 70s of the last century, the author developed special electronic equipment, using resonance at complex frequencies [5]. When developing the equipment, problems of the STR mentioned above weren't taken into account. And existence of such resonance discovered by the author was the evidence of physical reality of imaginary numbers. He even was granted some patents for equipment using such a resonance [10] [11].

Therefore, in contrast to the STR, in which the denial of physical reality of imaginary numbers was postulated and unconvincingly substantiated, the statement about physical reality of imaginary numbers was proved both theoretically and experimentally.

In the course of further research some more evidence was found. For example, this principle can persuasively be proved by means of Ohm's law [5] [6], discovered in 1826 and known to all educated people (but still not fully realized physically).

Indeed, according to the interpretation of the law [12] proposed Charles Proteus Steinmetz (1865-1923) in1897, not only resistors, but also capacitors and inductors have AC resistance. Moreover, although resistance of the latter is measured by imaginary numbers, they are measured by devices that are now available in any electrical and electronic laboratories. Consequently, the so-called imaginary inductive and capacitive resistance is physically real.

After all, what a proof of physical reality of concrete imaginary numbers can be more convincing than the opportunity to register the existence of this imaginary (only in mathematical sense) physical entity by instruments? In fact, we have found confirmation of existence of radioactivity, X-ray, magnetic field, infrasonic and ultrasound, radio waves, electric current and many other physical entities imperceptible to human senses, using instruments.

Albert Einstein argued: "Truth is what stands the test of experience".

Max Planck was of the same opinion: "An experiment is a question which science poses to nature and measurement is the recording of nature's answer". 
However, understanding of physical nature of a variety of real physical entities measured by imaginary numbers (for example, those corresponding to the Euler's formula) is a new and very complex problem in physics, which would take too long to completely resolve it. However, it has already been solved in the theory of electric circuits. Further, it is also shown how it has been solved in astrophysics.

\section{Adjustment of the Theory of Special Relativity Given the Principle of Physical Reality of Imaginary Numbers. Hidden Multiverse}

Since, as it turned out, the STR was wrong in its denial of physical reality of imaginary numbers, it has actually to be adjusted [13]. This requires that physical nature of imaginary mass in Formula (1) Einstein-Lorentz, as well as imaginary time and other imaginary physical quantities are to be explained in other relativistic formulas. That is, we should to do what authors of the STR could not do a hundred years ago.

The explanation is as follows. Physical objects moving at sub-light speeds, for example, tachyons [14] [15], are outside the event horizon. This means that they are invisible to us because they move away from us faster than their light beam approaches us. Since, according to Formula (1) and the rest of the relativistic formulas, physical objects moving at speed $v>c$ have parameters measured by imaginary numbers, and according to the principle of physical reality of imaginary numbers they actually physically exist, they do not exist in our universe. Consequently, they exist in a different universe. This invisible universe can be for certainty referred to as a tachyon. In other words, it is a physical representation of imaginary numbers in astrophysics. Similarly, our universe may be called a tardyon by the name of elementary particles moving at sub-light speed. Our universe is, therefore, a physical representation of real numbers in astrophysics. Consequently, the conclusion is that there is rather a multiverse physically existent, than a monoverse, as is still believed by supporters of the current incorrect version of the STR. And since the multiverse is invisible to us, it is, unlike many other hypothetical multiverses, called a hidden multiverse [16] [17].

\section{Extra Dimensions}

In this case it is assumable that tachyon universe is also inhabited and its inhabitants perceive their universe just as human beings perceive their universe, i.e., tachyon universe have the same physical, chemical and other laws that operate in our universe. Or, in other words, according to the first postulate of the STR it corresponds to the concept of inertial reference system.

However, it can be seen from the graph shown in Figure 1(a) that Formula (1) does not meet this condition, as fragments of the graph are different at $v<c$ and at $v>c$. Therefore we adjust this formula as follows:

$$
m=\frac{m_{0} i^{q}}{\sqrt{1-\left(\frac{v}{c}-q\right)^{2}}}=\frac{m_{0} i^{q}}{\sqrt{1-\left(\frac{w}{c}\right)^{2}}}
$$


where $q=\lfloor v / c\rfloor$ is the "floor" function (its graph is shown in Figure 2(a)) of argument $v / c$;

$w=v-q c$ is the local velocity for each universe (its graph is shown in Figure 2(b)), which can take values only in the range $0 \leq w<c$;

$v$ is the velocity measured from our tardyon universe, which, therefore, can be called a tardyon velocity.

Other relativistic formulas of the STR can be adjusted in a similar manner.

Graph of Formula (2) is shown in Figure 1(b). As can be seen, the parameter $q=0$ on this graph corresponds to our tardyon universe and the parameter $q=1$ corresponds to the tachyon universe. However, the value $q$ in Formula (2) can take on (and, as shown below, does take on) greater values. Therefore, its:

- parameter $q=2$ corresponds to tardyon antiverse (containing antimatter, since its mass, time and other physical quantities are real and negative);

- parameter $q=3$ corresponds tachyon antiverse (containing another antimatter, since its mass, time and other physical quantities are imaginary and negative);

- parameter $q=4$ corresponds to a different tardyon universe (containing the same matter as in our universe, since its mass, time, and other physical quantities are real and positive);

- parameter $q=5$ corresponds to another tachyon universe (containing the same "another" matter as in our universe, corresponding to $q=1$, since its mass, time and other physical quantities are imaginary and positive) etc.

The above-specified order of mutual spatial position of parallel universes prevents the annihilation of matter and antimatter of both tardyon and tachyon universes.

Formula (2) becomes more comprehensive, if its value $q$ is considered as an independent variable, which is an extra dimension of a hidden multiverse. Hence, it can be concluded that all these universes exist in different dimensions [18].

\section{Portals}

The universes existing in different dimensions can be called parallel, because, despite its infinity, they never intersect each other. However, floating in multidimensional space

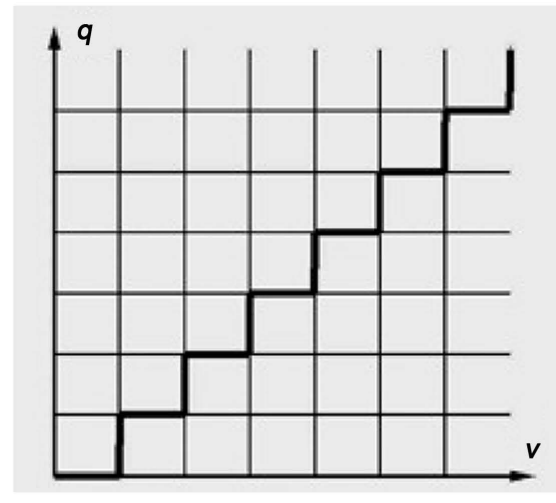

(a)

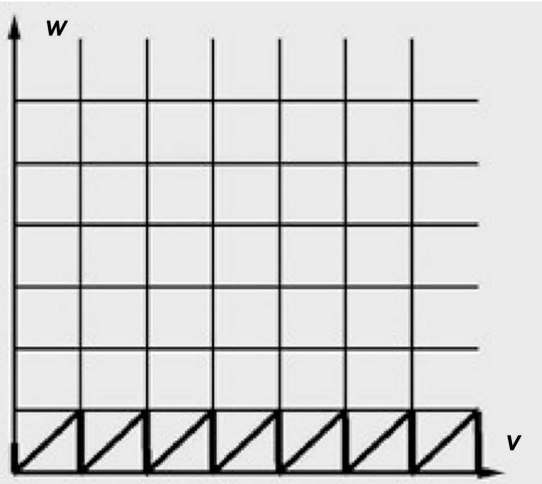

(b)

Figure 2. The graph of functions $q(v)$ and $w(v)$. 
they permanently or occasionally touch each other in many spots and even penetrate into each other, forming some transition zones referred to as portals or star gates [19]. There are plenty of them on Earth (and so much the more outside the Earth). On Earth they are the so-called anomalous zones.

\section{Dark Matter and Dark Energy. Adjustment of the Structure of Hidden Multiverse}

In the 20th century (at different times) some very strange objects were discovered in astrophysics. They have been called dark matter and dark energy [20] [21] due to their obscurity and full transparency. Since no chemical element known to us has been found in their composition, and their total weight has been over twenty times greater than the mass of all the objects of a visible universe, even modern understanding of the term 'matter' causes doubts. And further very intensive long-term study of dark matter and dark energy phenomena has not brought scientists closer to the understanding of its essence.

Therefore, Albert Einstein's remark is quite applicable to the current situation: "Insanity: doing the same thing over and over again and expecting different results".

Confucius was of the same opinion: "The hardest thing of all is to find a black cat in a dark room, especially if there is no cat".

This has turned out to be the case. These astrophysical objects have appeared to be so inexplicable only because of the incorrect formulation of task aimed at explanation in full compliance within the existing incorrect version of the STR.

In fact, these objects are invisible parallel universes (except our universe) of the hidden multiverse. Besides, they are an experimental proof of the hidden multiverse which has the following parameters, calculated according to the data obtained by the WMAP [22] and Planck [23] space stations [24]-[26]:

- the hidden multiverse contains about 20 ... 22 parallel universes;

- among them 6 other parallel universes, which together form what astrophysicists call dark matter, are adjacent to our universe;

- other parallel universes, which are more distant from us, together form what astrophysicists call dark energy;

- all parallel universes are interconnected by numerous unidirectional and bidirectional portals;

- in addition, some of the parallel universes of our hidden multiverse are connected by portals with one, two or three other multiverses (observable neither by electromagnetic, nor by gravitational manifestations), which together form a supermultiverse;

- besides, chemical composition of any universe cannot be determined from another universe.

Given these results, the structure of the multiverse described above has been verified. And it turned out that it complies with the principle of physical reality of one of variants of hypercomplex numbers called quaternions [27], containing three imaginary 
units $i_{1}, i_{2}, i_{3}$, rather than of complex numbers. Lorentz-Einstein formula corresponding to the structure appeared to be even more complicated

$$
m=\frac{m_{0}\left(i_{1}\right)^{q}\left(i_{2}\right)^{r}\left(i_{3}\right)^{s}}{\sqrt{1-[v / c-(q+r+s)]^{2}}}=\frac{m_{0}\left(i_{1}\right)^{q}\left(i_{2}\right)^{r}\left(i_{3}\right)^{s}}{\sqrt{1-(w / c)^{2}}}
$$

It uses the same designations as in Formula (2).

It follows from Formula (3), that the hidden multiverse has actually three additional dimensions $q, r, s$, rather than one, as previously assumed in analysis of the Formula (2). The total number of spatial dimensions in the hidden multiverse is equal to six: three dimensions $x, y, z$ are spatial coordinates within each parallel universe, and another three dimensions $q, r, s$ define the position of all parallel universes (see Figure 3) in multidimensional space of the multiverse.

In view of this, the hidden multiverse can have the structure shown in Figure 4 [18].

Consequently, the existing version of the STR applies only to our tardyon universe, whereas the adjusted version of the STR applies to the entire hidden multiverse. Thus, the principle of light speed non-exceedance turned out to be unnecessary in the adjusted STR.

It should be noted to the credit of Albert Einstein that he did not excluded such adjustment in the future. He wrote: "No single idea, which I would be sure that it will stand the test of time".

\section{Evidence of Existence of the Hidden Multiverse}

Apparently it can be proved. That is, unlike numerous other multiverse hypotheses [28]-[38], which are unverifiable (the existence of which will never be neither confirmed nor refuted), the concept of the hidden multiverse is completely verifiable. In fact, existence of the hidden multiverse:

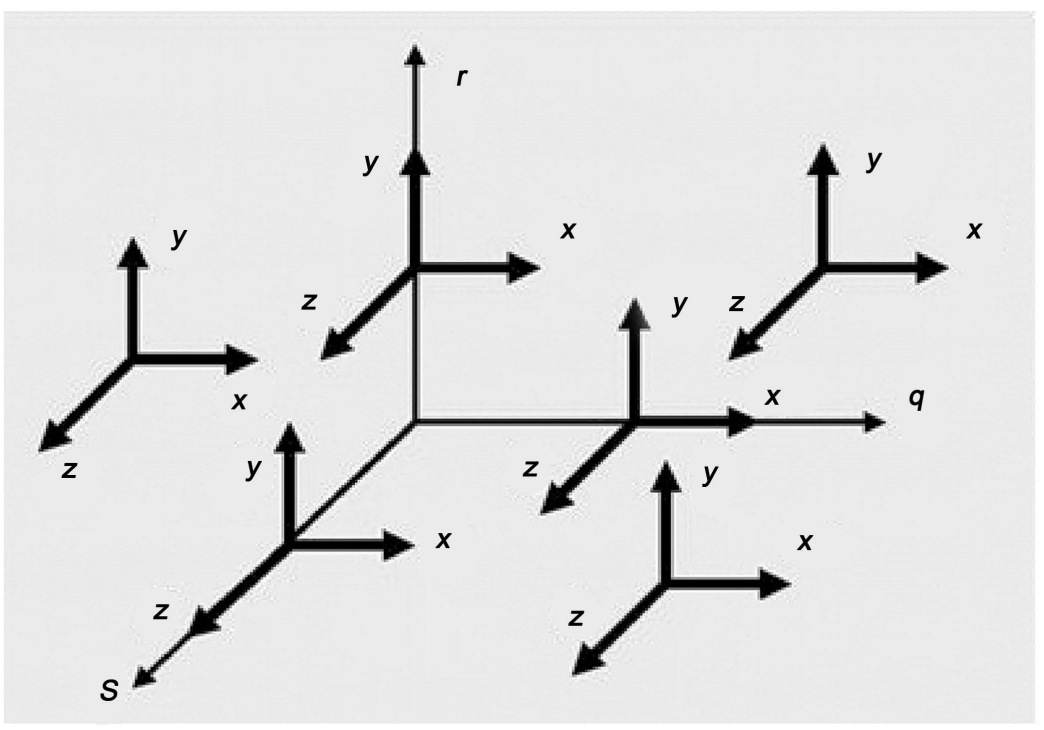

Figure 3. The six-dimensional space of the hidden multiverse. 


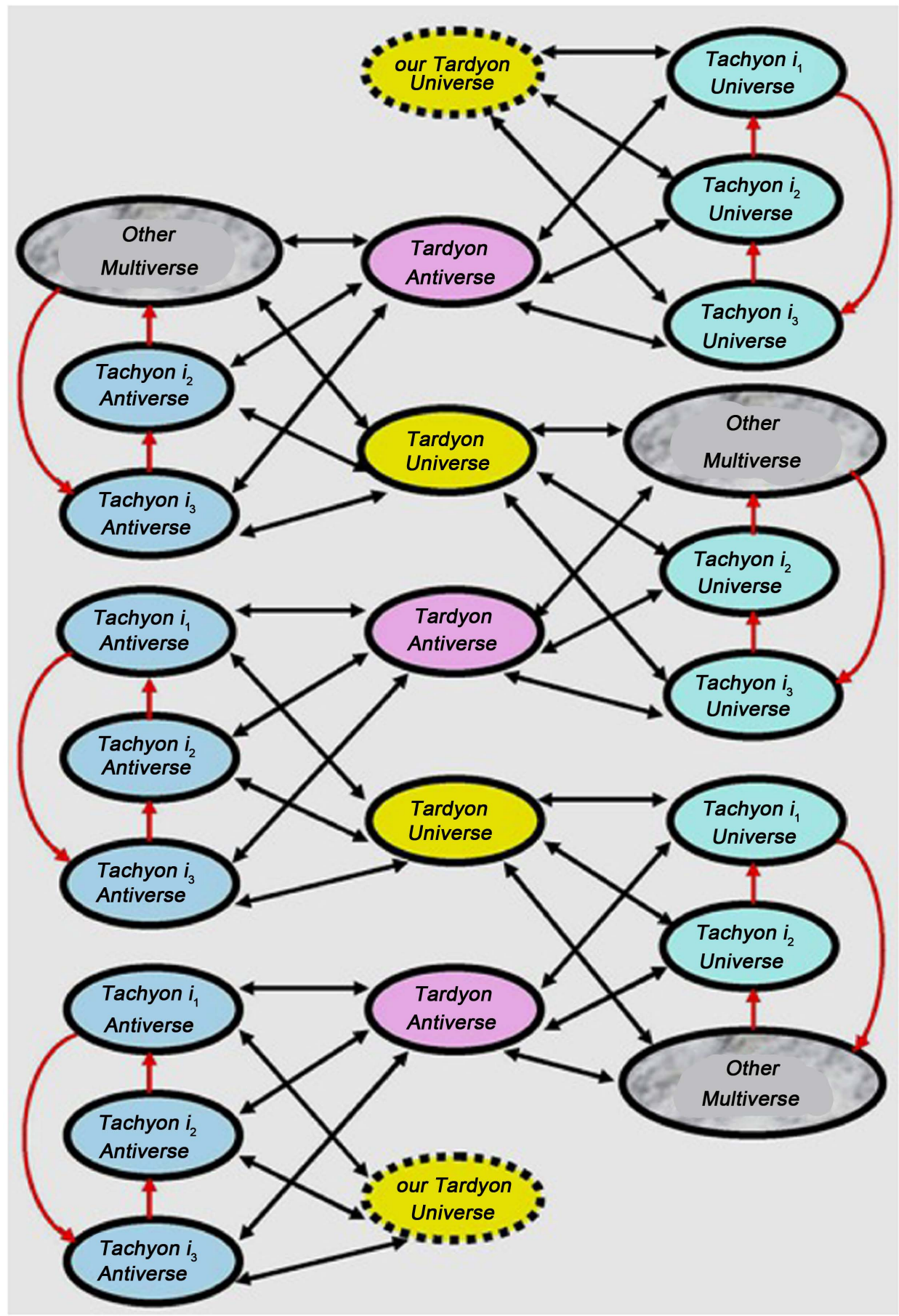

Figure 4. Supposed structure of the hidden multiverse.

- firstly, is proved by the phenomena of dark matter and dark energy;

- secondly, can be proved by discovering portals and visiting neighbors of parallel universes through them;

- thirdly, can be proved by the experiments at the Large Hadron Collider and other accelerators, resulting in the occurrence of tachyons. 


\section{Conclusions}

Thus, existence of the hidden multiverse, unlike other hypothetical multiverses, can be considered proven.

Practical discovery of the hidden multiverse and establishing contacts with inhabitants of other parallel universes [39] (which are likely much more intellectually developed and powerful) would considerably surpass Columbus' discovery of America by its consequences for human civilization.

\section{References}

[1] Grillo, G. and Einstein, A. (1920) Relativity: The Special and General Theory. H. Holt and Company, New York.

[2] Hawking, S.W. and Penrose, R. (2010) The Nature of Space and Time. Princeton University Press, Princeton \& Oxford. http://dx.doi.org/10.1515/9781400834747

[3] Antonov, A.A. (2010) General Mathematics Notes, 1, 11-16. http://dx.doi.org/10.17686/sced_rusnauka_2010-887

[4] Antonov, A.A. (2015) General Mathematics Notes, 31, 34-53.

[5] Antonov, A.A. (2015) American Journal of Electrical and Electronics Engineering, 3, 124129.

[6] Antonov, A.A. (2015) Global Journal of Physics, 2, 145-149.

[7] Popper K.R. (1972) Conjectures and Refutations. The Growth of Scientific Knowledge. Routledge and Kegan Paul, London and Henley.

[8] Cialdini, R.B. (2008) Influence: Science and Practice. 5th Edition, Pearson, US.

[9] Antonov, A.A. (2014) Global Journal of Science Frontier Research A: Physics and Space Science, 14, 51-59. http://dx.doi.org/10.17686/sced_rusnauka_2014-901

[10] Antonov, A.A. and Bazhev, V.M. (1970) Means of Rising Deflecting Currents for Spiral Beam Sweep on the CRT Screen. Patent of USSR \# 433650.

[11] Antonov, A.A. (1977) Output Scanning Stage. Patent of USSR \# 879618.

[12] Steinmetz, C.P. (2010) Theory and Calculation of Electric Circuits. Nabu Press. Charleston.

[13] Antonov, A.A. (2014) American Journal of Scientific and Industrial Research, 5, 40-52.

[14] Tanaka, S. (1960) Progress of Theoretical Physics (Kyoto), 24, 171-200. http://dx.doi.org/10.1143/PTP.24.171

[15] Feinberg, G. (1967) Physical Review, 155, 1089-1105. http://dx.doi.org/10.1103/PhysRev.159.1089

[16] Antonov, A.A. (2011) British Journal of Science, 2, 51-60. http://dx.doi.org/10.17686/sced_rusnauka_2011-892

[17] Antonov, A.A. (2015) International Journal of Advanced Research in Physical Science, 2, 25-32. http://dx.doi.org/10.17686/sced_rusnauka_2015-903

[18] Antonov, A.A. (2016) Philosophy \& Cosmology, 6, 11-27.

[19] Antonov, A.A. (2016) Journal of Modern Physics, 7, 1228-1246. http://dx.doi.org/10.4236/jmp.2016.710111

[20] Freeman, K. and McNamara, G. (2006) In Search of Dark Matter. Springer, New York.

[21] Nicolson, I. (2007) Dark Side of the Universe: Dark Matter, Dark Energy, and the Fate of the Cosmos. Johns Hopkins University Press, Baltimore. 
[22] Hinshaw, G., Larson, D., Komatsu, E., et al. (2013) Nine Year Wilkinson Anisotropy Probe (WMAP) Observations: Cosmological Parameter Results. arXiv: 1213.5226 [astro-ph/CO].

[23] Adam. R., Ade, P.A.R., Aghanim, N., et al. (2015) Plank 2015 Results. 1. Overview of Products and Scientific Results. arXiv: 1502.01582v2 [astro-ph.CO].

[24] Antonov, A.A. (2015) American Journal of Modern Physics, 4, 180-118. http://dx.doi.org/10.11648/j.ajmp.20150404.14

[25] Antonov, A.A. (2015) Global Journal of Science Frontier Research A: Physics and Space Science, 15, 33-38.

[26] Antonov, A.A. (2015) Cosmology, 19, 40-61.

[27] Kantor, I.L. and Solodovnikov, A.S. (1989) Hypercomplex Numbers. Springer Verlag, Berlin.

[28] Deutch, D. (1998) The Fabric of Reality: The Science of Parallel Universes and Its Implications. Penguin Books, New York.

[29] Greene, B. (2000) The Elegant Universe: Superstrings, Hidden Dimensions, and the Quest for the Ultimate Theory. Random House Inc., New York.

[30] Kaku, M. (2006) Parallel Worlds: A Journey through Creation, Higher Dimensions, and the Future of the Cosmos. Doubleday, New York.

[31] Steinhardt, P.J. and Turok, N. (2007) Endless Universe: Beyond the Big Bang. Doubleday, New York.

[32] Vilenkin, A. (2007) Many Worlds in One: The Search for Other Universes. Hill \& Wang, New York.

[33] Weinberg, S. (2008) Cosmology. Oxford University Press, New York.

[34] Carr, B. (Ed.) (2009) Universe or Multiverse? Cambridge University Press, Cambridge.

[35] Lucash, V.N. and Mikheyeva, E.V. (2010) Physical Cosmology. Physmathlit, Moscow.

[36] Greene, B. (2011) The Hidden Reality: Parallel Universes and the Deep Laws of the Cosmos. Random House Inc., New York.

[37] Deutsch, D. (2012) The Beginning of Infinity: Explanations That Transform the World. Reprint Edition, Penguin Books, New York.

[38] Tegmark, M. (2015) Our Mathematical Universe: My Quest for the Ultimate Nature of Reality. Vintage, New York.

[39] Antonov, A.A. (2015) Where to Look for Alien Civilisations. Cosmology. Commentaries: Stephen Hawking's Aliens. The Search for Intelligent Extraterrestrial Life, Project Breakthrough Listen. 
Submit or recommend next manuscript to SCIRP and we will provide best service for you:

Accepting pre-submission inquiries through Email, Facebook, LinkedIn, Twitter, etc. A wide selection of journals (inclusive of 9 subjects, more than 200 journals)

Providing 24-hour high-quality service

User-friendly online submission system

Fair and swift peer-review system

Efficient typesetting and proofreading procedure

Display of the result of downloads and visits, as well as the number of cited articles

Maximum dissemination of your research work

Submit your manuscript at: http://papersubmission.scirp.org/

Or contact jmp@scirp.org 